\title{
Diabetes Mellitus: desafios relacionados ao autocuidado abordados em Grupo de Apoio Psicológico
}

\author{
Diabetes Mellitus: challenges related to self-care adressed in a Psychological Support Group \\ Diabetes Mellitus: desafíos relacionados con el autocuidado en Grupo de Apoyo Psicológico
}

\author{
Nunila Ferreira de Oliveira', Maria Conceição Bernardo de Mello e Souza', \\ Maria Lúcia Zanetti', Manoel Antônio dos Santos" \\ 'Universidade de São Paulo. Escola de Enfermagem de Ribeirão Preto, SP \\ "Universidade de São Paulo. Faculdade de Filosofia, Ciências e Letras de Ribeirão Preto, SP
}

Submissão: $16 / 01 / 2010$

Aprovação: 20/06/2010

\section{RESUMO}

O objetivo foi identificar os sentimentos dos usuários associados ao diagnóstico do Diabetes Mellitus (DM), as dificuldades inerentes ao controle glicêmico e estratégias de enfrentamento adotadas diante das demandas impostas pela doença. Estudo Qualitativo realizado com grupo de pessoas acompanhadas por eQuipe multiprofissional de um centro de pesquisa e extensão universitária de Ribeirão Preto, SP, Brasil. Quatro sessões grupais Que constituíram o corpus da pesQuisa, foram audiogravadas e transcritas. A análise temática originou as categorias: sentimentos despertados a partir do diagnóstico do DM; dificuldades no controle da doença e estratégias de enfrentamento. Os resultados evidenciaram diferentes modos de lidar com os desafios suscitados pela doença no cotidiano, Que influenciam diretamente na adesão ao tratamento e constituem elementos relevantes para o planejamento de intervenções mais efetivas.

Descritores: Saúde Mental; Diabetes Mellitus; Processos grupais; Enfermagem.

\section{ABSTRACT}

The purpose was to identify the feelings of users associated with the diagnosis of Diabetes Mellitus (DM), the difficulties inherent of glycemic control and coping strategies adopted in face of the demands imposed by the disease. Qualitative study carried out with patients followed by a multiprofessional team group in a research center from a university in Ribeirão Preto, SP, Brazil. Four group sessions, that were audio recorded and transcribed, constituted the corpus of research. The thematic analysis led to the categories: feelings aroused from the diagnosis of DM; difficulties in controlling the disease and coping strategies. The results showed different ways of dealing with the challenges posed by the disease on the diary activities, that directly influence on the treatment adherence and factors which are relevant for the design of more effective interventions.

Key words: Mental health; Diabetes Mellitus; Group processes; Nursing.

\section{RESUMEN}

El objetivo fue identificar los sentimientos de los usuarios asociados con el diagnóstico de Diabetes Mellitus (DM), las dificultades inherentes al control de la glucemia y las estrategias adoptadas en la cara de las exigencias impuestas por la enfermedad. Estudio cualitativo realizado con un grupo de personas atendidas por el equipo multidisciplinario en un centro de investigación y extensión de la universidad en Ribeirão Preto, SP, Brasil. Fueron audio grabadas y transcritas cuatro sesiones de grupo, Que constituye el corpus de la investigación. El análisis temático dirigido a las categorías: sentimientos que a partir del diagnóstico de la DM, las dificultades en el control de la enfermedad y las estrategias de afrontamiento. Los resultados muestran diferentes maneras de lidiar con los desafíos planteados por la enfermedad los procesos Que influyen directamente en la adherencia al tratamiento y los factores que son relevantes para el diseño de intervenciones más eficaces.

Descriptores: Salud mental; Diabetes Mellitus; Processos grupais; Enfermería.

AUTOR CORRESPONDENTE Nunila Ferreira de Oliveira. Rua Tenente Catão Roxo, 1359- apto 34. CEP 1405 1-140. Ribeirão Preto, SP.

E-mail: nunila@usp.br 


\section{INTRODUÇÃO}

Atualmente, vive-se um descompasso entre o perfil epidemiológico da população e o foco da assistência em saúde. Enquanto as condições crônicas prevalecem, as ações de saúde são essencialmente curativas, voltadas para o atendimento de agravos agudos. Para responder a essa demanda é necessário priorizar ações relacionadas com a promoção da saúde e prevenção de complicações ${ }^{(1-}$ 4). Intervenções educativas são essenciais nesse contexto de atendimento, com vistas à inclusão das pessoas no cuidado da própria saúde.

Dentre as condições crônicas, o Diabetes Mellitus (DM) é uma morbidade relevante devido ao acometimento populacional crescente e à natureza incapacitante dos agravos decorrentes de suas complicações. As intervenções profissionais visam, especialmente, à obtenção do controle glicêmico para evitar ou minimizar tais complicações ${ }^{(5-6)}$.

Entretanto, o controle glicêmico constitui um desafio na medida em Que depende da mudança de hábitos, muitas vezes arraigados culturalmente e associados a Questões subjetivas relacionadas ao modo de vida das pessoas. A troca de experiências entre as pessoas Que convivem com DM é relevante para Que possam repensar as ações cotidianas dos cuidados direcionados à doença ${ }^{(7)}$.

Nesse sentido, o atendimento em saúde deve incluir atividades de cunho educativo e também de suporte para apoiar a população no enfrentamento dos desafios inerentes ao tratamento do DM. Assim, "habilidades avançadas de comunicação, técnicas de mudança de comportamento, educação do paciente e habilidades de aconselhamento são necessárias para auxiliar os pacientes com problemas crônicos"(I).

Estudos $^{(7-9)}$ demonstram o potencial do trabalho em grupo para o atendimento de pessoas com DM, na medida em Que a interação e identificação com outras pessoas Que convivem com a mesma doença podem auxiliar no processo do autocuidado.

Diante do exposto, o presente estudo foi conduzido em um Centro de Pesquisa e Extensão Universitária (CPEU), no qual uma equipe multiprofissional oferece acompanhamento em grupo a pessoas com DM, com vistas à educação em saúde voltada ao fortalecimento do autocuidado. Desse modo, este estudo teve por objetivo identificar os sentimentos associados ao diagnóstico do DM, as dificuldades inerentes ao controle glicêmico e as estratégias de enfrentamento adotadas pelos participantes do grupo diante das demandas impostas pela doença.

Explorar aspectos relacionados ao tratamento do DM a partir da ótica de pessoas com essa condição crônica pode oferecer subsídios para o planejamento de intervenções mais congruentes com as particularidades Que interferem na adesão ao autocuidado.

\section{MÉTODO}

Estudo Qualitativo realizado junto ao CPEU. Esse serviço está situado em uma cidade do interior paulista e oferece acompanhamento a pessoas com DM visando ao desenvolvimento de autonomia para o autocuidado.

Nos meses de fevereiro e agosto de cada ano, após divulgação junto à população, por meio de jornais impressos e programas de televisão, é feito o cadastramento das pessoas interessadas. O acom- panhamento acontece ao longo do semestre, os encontros são semanais, com duração aproximada de três horas, constando de atendimento em grupo, seguido de avaliação de parâmetros clínicos como: peso corporal, glicemia capilar pós-prandial, pressão arterial e circunferência abdominal.

O CPEU conta com uma equipe multiprofissional formada por enfermeiros, psicólogos, educadores físicos e nutricionistas, Que por sua vez se subdividem em Quatro equipes, conforme as especialidades: Enfermagem, Saúde Mental, Educação Física e Nutrição, Que coordenam os atendimentos em grupo. Cada equipe atende o grupo uma vez por mês, segundo as suas especificidades. Para assegurar a efetivação de um trabalho interdisciplinar, a equipe multiprofissional se reúne semanalmente, durante uma hora, para discutir o andamento das atividades.

A coleta de dados foi realizada de agosto a dezembro de 2008, durante as Quatro sessões do grupo conduzido pela equipe de Saúde Mental. As sessões grupais foram coordenadas por uma enfermeira, mestranda em Enfermagem Psiquiátrica, e um psicólogo. Esses profissionais participaram integralmente das atividades realizadas no CPEU, atuando também como coordenadores do grupo.

Esse atendimento visa a oferecer apoio para as demandas emocionais dos participantes, relacionadas com os desafios cotidianos inerentes aos cuidados dispensados ao DM. Durante as atividades do grupo eram propostas técnicas disparadoras pouco estruturadas, Que incentivavam a participação dos integrantes do grupo e possibilitavam a abordagem e discussão de temas referentes às demandas dos participantes frente ao DM e seu tratamento.

Participaram da pesquisa 16 pessoas, componentes do grupo. As sessões foram audiogravadas, mediante anuência de todos os participantes e, posteriormente, transcritas na íntegra, constituindo o corpus de análise.

Os dados foram submetidos à análise temática de conteúdo ${ }^{(10)}$, seguindo as seguintes etapas: pré-análise, Quando se realizou a leitura das transcrições das sessões grupais, buscando maior contato com o conteúdo dos dados; exploração do material, com leitura aprofundada e repetitiva com vistas a identificar as unidades de registro, bem como classificar e codificar os dados para a conformação de categorias; e, finalmente, o tratamento e interpretação das categorias obtidas.

Para a sistematização dos resultados, foram selecionados relatos nos Quais os participantes utilizaram o espaço do grupo para discorrer sobre suas trajetórias de vida desde o diagnóstico do $\mathrm{DM}$, incluindo os sentimentos relacionados à doença, as dificuldades encontradas e estratégias de enfrentamento adotadas. Com vistas a preservar o anonimato, ao longo da apresentação dos resultados e sua discussão, os participantes da pesquisa foram referidos pela letra $\mathrm{P}$, seguida de um número de identificação. As falas passaram por correções gramaticais com vistas a torná-las mais claras e compreensíveis.

Quanto aos aspectos éticos, foram seguidas as diretrizes preconizadas para pesQuisas com seres humanos pela Resolução 196/ 96 do Conselho de Nacional de Saúde ${ }^{(1)}$, com a aprovação do projeto pelo Comitê de Ética da EERP/USP, em reunião do dia 19 de dezembro de 2007, protocolo ${ }^{\circ}$ 0849/2007. Os participantes, após terem sido orientados acerca dos objetivos da pesquisa, assinaram o Termo de Consentimento Livre e Esclarecido. 


\section{RESULTADOS E DISCUSSÃO}

Com vistas a responder aos objetivos Que nortearam a execução do presente estudo, a partir da análise temática das trancrições das sessões grupais foram elaboradas três categorias: Sentimentos despertados a partir do diagnóstico do DM, Dificuldades no controle do DM e Estratégias de enfrentamento. Os resultados serão apresentados a seguir, juntamente com as falas Que deram sustentação para as discussões e inferências.

\section{Sentimentos despertados a partir do diagnóstico do DM}

Durante as sessões do grupo, os relatos referentes à experiência do diagnóstico do DM foram acompanhados da rememoração dos sentimentos vivenciados naQuele período. As falas seguintes são representativas dos diferentes sentimentos compartilhados no grupo acerca desse marcante momento na vida de cada participante:

\section{[...] fiquei nervoso com aquilo... Eu não aceitei [...] (PI I)}

[...] fiquei revoltado, porQue é uma coisa Que você não espera na vida $[. .].(\mathrm{P} 4)$

[...] fiquei triste, porém satisfeito. Porque poderia ser uma doença terminal... Essa não tem cura, mas não é terminal. (P7)

Pra mim, uma doença QualQuer [...] o começo para mim foi igual Quando você está resfriado... vai fazer tratamento e passa, entendeu? ...e eu estava com 720 de glicemia [...J (P9)

As falas apontam diferentes reações frente ao diagnóstico. Dentre os Que apresentaram reações emocionais negativas, prevaleceram sentimentos disfóricos como raiva e tristeza, levando a reações de contestação e depressão, respectivamente. Outros buscaram minimizar o efeito devastador da experiência, utilizando-se de eufemismos, ao não se preocuparem com o diagnóstico do DM por não conhecerem a doença e suas possíveis consequências. A possibilidade de narrar a própria situação emocional diante do adoecimento, e também de perceber diversas reações a partir de relatos dos outros participantes do grupo, podem contribuir para novas interpretações e explicações para o processo de adoecer, indicando diferentes formas de enfrentamento da situação ${ }^{(12)}$.

Quando traçamos um paralelo entre as diferentes reações dos participantes frente ao diagnóstico do DM e a necessidade de Que todos, cada Qual com suas especificidades, envolvam-se no tratamento, percebemos o desafio Que é colocado para os serviços de saúde. Há necessidade de se disponibilizarem intervenções coerentes e diversificadas, visando a assegurar a adesão ao tratamento. Para tanto, além de fornecer informações, é interessante Que a equipe de saúde ofereça acompanhamentos mais próximos, pormenorizados e com foco no posicionamento assumido por cada indivíduo frente à condição de adoecimento, a relação mantida com o processo de adoecer e os modos de lidar com as exigências do tratamento ${ }^{(13)}$.

A ponderação entre padronizar e/ou individualizar intervenções merece destaque nas ações em saúde de cunho eminentemente educativo, visando sempre a um incremento da adesão terapêuti$\mathrm{Ca}^{(2)}$, considerando as diferenças existentes entre as formas de lidar com a doença, conforme nos indicam os relatos Que sustentam a presente categoria.

\section{Dificuldades no controle do DM}

Uma das características marcantes das condições crônicas em geral é o espaço que, a partir do diagnóstico, a doença passa a ocupar na vida das pessoas. Os relatos de alguns participantes sugerem Que eles não conseguem lidar com essa condição e simplesmente negam a existência da doença:

[...] a pior doença para mim foi o diabetes, então eu comecei a encarar como se eu não tivesse ele, eu só tomo café com açúcar, eu como doce depois do almoço... E eu tô vivendo bem... Então eu me sinto melhor hoje. (PI0)

\section{[...] eu prefiro mais achar Que eu não tenho nada dessas coisas $[\ldots]$ (P5)}

Paradoxalmente, esses participantes buscaram um grupo de apoio para pessoas com DM de forma espontânea, o Que sugere Que há uma ambivalência entre o sentimento de negação da condição e a percepção da necessidade de ampliação de sua consciência sobre o manejo do tratamento.

No contexto da convivência com o DM, a necessidade de mudança de hábitos simboliza fortemente a relação com a doença. Usualmente, aceitar a necessidade dessa mudança é fonte de dificuldades relatadas no grupo, como podemos perceber nas falas a seguir:

"[...] Quando a gente descobre Que tem a bendita, a vida da gente muda bastante... Você perde todo o caminho que você tinha antes, tem Que voltar para trás. (P4)

O relato acima nos indica Que incorporar uma nova forma de viver, diferente da norma construída pelo indivíduo ao longo dos anos, parece ser um processo doloroso por implicar renúncias e necessidade de formular readaptações no viver.

A adaptação do paciente frente ao diagnóstico do DM impõe a criação gradativa de uma identidade relacionada com a doença ${ }^{(8)}$. Porém, tendo-se em vista a relação implícita do processo saúdedoença-cuidado com a forma como as pessoas percebem a realidade concreta, a construção de tal identidade parece ser dificultada pela própria característica silenciosa dos primeiros sintomas do DM, Que é uma condição na Qual o portador não se sente doente ${ }^{(14)}$.

Nesse sentido, uma "importante tensão dialética se apresenta entre uma doença Que não é assumida pelo doente e a necessidade de aproximar-se do serviço de saúde, devido aos riscos ameaçadores da vida"(14). Essa relação fica evidente nas falas dos participantes do presente estudo, Que buscaram o serviço por decisão própria e, no entanto, proferem relatos como os descritos anteriormente, Que denotam a negação da condição de adoecimento a ponto de negligenciarem o autocuidado, chegando a adotar hábitos de risco.

Os relatos descritos até aqui nos permitiram vislumbrar a complexidade inerente ao processo de mudança de hábitos. Nesse contexto, em seus relatos, os participantes do grupo enfatizaram a reeducação alimentar como o maior desafio: 
[...] eu não consigo seQuer fazer regime para perder peso, Que eu sei Que preciso. [...] Então eu fico pensando: se eu não consigo nem fazer regime, como Que eu vou conseguir o resto? (P4)

Pode juntar todo mundo para ver o Que é mais [difícil], se não é o regime alimentar. (PII)

\section{[...] a gente não pode comer muito mesmo, porQue já perdeu a} saúde. [...] (PI6)

Os relatos acima transcritos indicaram Que alguns participantes percebem o controle alimentar como um martírio, considerando-o como uma meta de tratamento difícil de ser concretizada. Assim, exibem reações Que podem variar desde a raiva e inconformismo diante das inúmeras "perdas" e restrições Que vivenciam em seu cotidiano durante o longo processo de adoecimento até sentimentos de descrença e culpa por transgredirem as restrições e recomendações preconizadas pela equipe de saúde. O estado emocional predominante é de fracasso e desmoralização, ao perceberem Que não conseguem se aproximar das metas Que gostariam de alcançar para preservar sua saúde.

É perceptível a angústia dos participantes Quando se referem à dificuldade de adaptação frente às necessidades impostas pela doença. Estudos também indicam a alimentação como um desafio relevante para as pessoas Que convivem com o DM, sendo apontadas dificuldades significativas de seguir as orientações dos profissionais $^{(15-16)}$

A discrepância entre os hábitos adotados pelos familiares e o restante da rede social e os hábitos alimentares "prescritos" pelos profissionais para a pessoa com DM foi um fator sinalizado como dificultador da adesão às metas da reeducação alimentar, como indicam as falas seguintes:

[...] na minha casa eu até prejudiquei o meu sogro, meu marido, meu filho, eu falei: olha, vocês não comprem pão aQui não; comprou, eu como! [...] (P5)

[...] eu é que tenho que me cuidar, porQue o meu marido fala assim: Come um pouQuinho [de doce], não faz mal! Eu falei: Não, não Quero, não posso comer doce, aí ontem, de tanto ele insistir, eu comi um pedaço de doce de abóbora. [...] (PI0)

[... se eu sair fora, igual agora, Que eu fieuei duas semanas, não tive condições de fazer tudo [reeducação alimentar] certinho... (P5)

Dentre os fatores Que influenciam a reeducação alimentar, o fator financeiro também foi mencionado como um dificultador relevante:

[...] isso atinge a maioria do povão, a parte econômica, porQue não é barato também você manter uma alimentação de acordo com o diabetes... E os testes, as fitas, tudo... a insulina, tudo caro, também é tudo caro. (P7)

[...] [mesa] farta com Qualidade, tem Que ter dinheiro. (...) se você for comprar o arroz integral. (P9)

Tais falas sinalizam a complexidade inerente à mudança dos hábitos alimentares, tendo-se em vista a diversidade de fatores envolvidos. Quando se pensa nos costumes alimentares de uma população, existem dificuldades de mudanças relacionadas a diversos fatores, tais como os hábitos condicionados, a rotina de horários, o valor cultural associado aos alimentos, além de Questões socioeconômicas ${ }^{(15)}$

Podemos apontar os modelos teóricos direcionados para condições crônicas como possibilidades de direcionamentos mais assertivos das intervenções implementadas nesse contexto. Por exemplo, o Modelo Transteórico, Que propõe estágios para o alcance da mudança de comportamentos, incluindo a possibilidade de recaídas, mostrou-se eficaz em outro estudo para direcionar intervenções visando à mudança de hábitos alimentares ${ }^{(17)}$.

As orientações profissionais para as pessoas Que convivem com o DM condicionam uma nova rotina de cuidados diários relacionados com a doença, como a prática de atividade física, o monitoramento dos níveis glicêmicos e o exame dos pés ${ }^{(5,6)}$. Nesse contexto, os participantes do grupo também referem dificuldades, como sinalizam os relatos a seguir:

[...] tem I4 dias que eu não venho fazendo o teste... é Que eu estou numa tensão muito grande [...] (P2)

Ficar olhando o pé para saber se não está inflamado, para saber se não vai cortar o pé, é triste... A realidade é triste demais. (PI)

Quando a diabetes estava alta, eu ficava andando na cidade, ia longe a pé, e não comia muito e estava com a diabete alta. (P6)

Os relatos permitiram conhecer a forma com Que os participantes do grupo lidam com as demandas de cuidados referentes ao DM. O conhecimento interfere de forma significativa nos comportamentos de autocuidado ${ }^{(17)}$, bem como na percepção Que os pacientes têm acerca da doença ${ }^{(8)}$.

Podemos perceber certa relativização do cuidado, com adoção de certas manobras compensatórias - Quando a glicemia está alta, pratica-se muita atividade física e reduz-se o consumo alimentar. Alguns percebem as atividades de autocuidado como prescrições a serem cumpridas e a referência aos cuidados é desvinculada da manutenção da saúde, mas ad@uire uma conotação mais ampla, como uma forma de "tratar" o DM.

Nas intervenções profissionais direcionadas a essa população é importante Que se estimule a percepção de Que tais hábitos são também buscados por pessoas com a intenção de alcançar um estilo de vida saudável, para que "pessoas diabéticas" se tornem "pessoas com diabetes", cujas vidas não precisam ter, necessariamente, o DM como foco centrall ${ }^{(8)}$.

Apesar do enfoque do presente estudo circunscrever-se ao DM, ao longo das sessões do grupo os participantes referiram Que, no curso de suas vidas, a doença não era o único - e às vezes nem o maior - desafio, como se pode observar nos relatos a seguir:

Tô tomando insulina, e até o começo do ano eu não tomava remédio pra diabetes, depois fui fazer tratamento pro vírus da 
Hepatite C. [...] (P8)

É porque eu já tenho a pressão alta, já tenho um mioma, e outras preocupações [...] (P)

...tem dia assim, Que bate aQuele negócio assim, Quer morrer, Quer morrer, é uma depressão... (P2)

Tais relatos indicam Que alguns fatores, como a presença de comorbidades, podem dificultar a adesão ao tratamento do DM. A associação entre DM e depressão é prevista em estudos ${ }^{(18-19)}$ Que demonstram Que essa condição crônica constitui um fator negativo relevante para a adesão ao tratamento e mudanças de hábitos. Dessa forma, durante os planejamentos dos cuidados em saúde deve-se atentar para a presença de comorbidades psicológicas, como a depressão e ansiedade, com vistas a buscar medidas terapêuticas efetivas.

Além dos problemas relacionados à saúde, também são descritas dificuldades associadas à família, problemas de perdas por morte, doenças e sobrecargas Que são igualmente relevantes na vida dos participantes do grupo:

[...] eu tenho um problema com a minha mãe, sabe? Essa doença, Alzheimer, é duro de aturar, viu? Porque tem hora que extrapola você também. [...] (P2)

PorQue eu vim de um trauma assim com a perda dos meus filhos e meu marido pirou com a perda deles e eu também tava entrando nessa [choro]... Da onde eu senti força para viver foi aqui. [...] (P2)

Em estudo realizado com grupo de mulheres com DM as participantes relataram sentir raiva de si mesmas por não conseguirem alcançar a almejada mudança de hábitos; foi constatada ainda Que a sobrecarga de responsabilidades perante os cuidados da família também é um fator Que contribui para a não efetivação dessas mudanças ${ }^{(18)}$.

Pelo próprio modus operandi do grupo de apoio, cuja execução era baseada nas demandas dos próprios pacientes, pode-se compreender de forma mais genuína as necessidades dos participantes, Que apesar de estarem engajados em um serviço direcionado para o apoio na terapêutica do DM, traziam consigo demandas de ordem emocional derivadas de outros problemas de saúde ou pessoais, próprios ou de pessoas do entorno familiar, Que influenciavam diretamente na adesão ao tratamento e, consequentemente, na obtenção do controle glicêmico ${ }^{(20)}$.

\section{Estratégias de enfrentamento}

$\mathrm{Na}$ análise dos dados, apesar das dificuldades relatadas, algumas falas demonstraram convivências relativamente positivas com o DM e maior capacidade de enfrentamento:

[...] tem Que aceitar tudo essas coisas, você aceitando fica mais fácil. [...] (PI2)

[...] se a gente não aceitar, a gente vai ficar sofrendo a vida inteira. [...] Então a gente tem Que aceitar e fazer tudo de me-
Ihor pra saúde da gente. (P2)

[...] já passei muitos bons momentos que, sendo diabética, estava totalmente controlada. (P3)

[...] saber Que tem o problema e saber conviver com ele [...] consequentemente, você tem qualidade de vida. [...] (P7)

A partir dos relatos coligidos, os participantes indicaram Que, apesar da crise que o diagnóstico do DM instaurou em suas vidas, o aprendizado de uma convivência saudável com a doença pode ser alcançado, seja por meio de uma aceitação passiva e conformista ou de um posicionamento mais ativo perante a doença e o tratamento, acreditando na possibilidade de reagir de forma mais serena às demandas emocionais e de agir de modo organizado para obtenção de melhor controle.

Intervenções mais efetivas no tratamento do DM são alcançadas Quando o problema das doenças crônicas não é contextualizado simplesmente como um sistema orgânico a ser monitorado ou um processo fisiológico, mas Quando são subsidiadas pelos contextos e condições individuais, Que influenciam na adesão ao tratamento $^{(2)}$.

Ao mesmo tempo que a mudança de hábitos é vista como uma dificuldade para alguns membros do grupo, outros buscam formas diferentes de enxergar o mesmo desafio. Com relação à reeducação alimentar, alguns membros do grupo referem:

\section{[...] o corpo da gente é outro, a disposição é outra [...] (P4)}

[...] eu estou me cuidando com aquilo que não pode [...] eu estou me sentindo bem [...] eu sinto que eu posso tudo, sem exagero. (P2)

Nesses relatos, podemos notar Que algumas pessoas conseguem identificar a mudança de hábitos alimentares como reeducação e, assim, buscam o equilíbrio entre vontade e moderação, percebendo essa mudança como positiva para suas vidas, ressaltando seus benefícios para o corpo e reconhecendo Que o descontrole pode ser prejudicial.

O relato abaixo demonstra uma estratégia compartilhada por um participante do grupo para a incorporação da atividade física como um hábito do cotidiano:

[...] eu não fazia caminhada... teve um dia que eu levantei mais cedo, [...] aí eu peguei o aparelhinho e fui medir, estava 160 a glicemia, falei: Quer saber, eu não vou tomar [medicamento] nada agora não, vou fazer uma caminhada, aí eu saí 40 minutos, Quando eu voltei, fui medir, foi pra 75 ... aí, agora, eu passei a fazer caminhada. (P8)

Mas eu ando sim, Quando a diabetes estava alta eu ficava andando na cidade, ia longe a pé, e não comia muito e estava com a diabete alta. [...] (PI6)

Tais relatos indicam que a atividade física, adotada após o diagnóstico do DM, passou a ser vista como uma prática positiva, porém o exercício físico é utilizado por alguns dos participantes 
como estratégia para baixar os níveis glicêmicos, ou seja, uma prática terapêutica. São poucos os Que referem os benefícios inerentes a essa prática, independentemente da sua capacidade de redução dos níveis glicêmicos.

Nesse sentido, a informação é uma ferramenta preciosa, tendose em vista Que, Quando as pessoas têm pouco conhecimento em relação à doença e à dinâmica do controle glicêmico, resistem a incorporar os comportamentos de autocuidado, o que dificulta a adesão terapêutica e, consequentemente, o controle dos níveis glicêmicos $^{(21)}$.

Os relatos ilustrativos da presente categoria indicaram Que alguns participantes do grupo alcançaram uma maior conscientização acerca do papel Que algumas atividades de autocuidado têm para a manutenção da saúde, inclusive reconhecendo na condição de adoecimento um estímulo para o autocuidado e uma oportunidade para a valorização da vida.

No contexto da convivência com o DM e as mudanças necessárias para o seu tratamento, percebemos, dentre os relatos, a presença da família como uma fonte de apoio no enfrentamento da doença no cotidiano. Os relatos seguintes denotam a importância Que tais vínculos representam para a subjetividade de cada participante Que convive com o DM, sendo Que alguns familiares conseguem ser participantes ativos nesse processo de autocuidado:

\section{[...] a família pega no pé com o que ela come, Quando ela come muito. (P5)}

[... d diminui bastante as coisas assim, então eu tento fazer o Que está pedindo, eu vejo pela minha mãe [Que também tem DM], ela tem 87 anos, ela não Quer morrer, ela fala Que não Quer. (PI2)

Eu, além de me cuidar, a minha esposa é Que nem um sargentão na minha casa, lá não sai fora do ritmo não, tem Que... ela vai em cima da letra ali e eu sou obrigado. (P4)

A família é considerada como fonte de apoio para a pessoa com $\mathrm{DM}^{(22)}$, no entanto, essa relação se torna tensa Quando o foco é a Questão da hereditariedade, a Qual indica Que a gênese da doença está relacionada à predisposição genética familiar ${ }^{(5)}$, conforme destado nos relatos a seguir:

A mãe tinha $[\mathrm{DM}]$ também, por isso que eu acho que é hereditário. (P7)

[...] o dia em que eu fiquei sabendo, eu fiquei muito triste e revoltada, e eu pensei: por Que só eu? Se eu tenho dois irmãos, por Que só eu? (PIO)

Por outro lado, a hereditariedade às vezes funciona como preparação subjetiva para a possibilidade de desenvolvimento futuro da doença, como nos indica o relato seguinte:

[...] eu, pra mim, como ela é hereditária, minha mãe é diabética, então não me pegou assim, de surpresa. [... (P7)

A estreita influência da família no processo de enfrentamento do DM também é um fator Que deve ser considerado nas intervenções direcionadas às pessoas acometidas. A tendência, Quando uma pessoa participa de um processo educativo em DM, é de Que o conhecimento obtido alcance também a família, podendo favorecer a adoção de hábitos saudáveis, tanto da parte do paciente Quanto de sua rede familiar. Esse processo pode influenciar de forma positiva a adesão ao tratamento e controle glicêmico ${ }^{(22)}$.

A convivência de alguns dos participantes com a doença é fortemente influenciada pelo medo das complicações futuras, o Que podem estimular a adoção de práticas de autocuidado. Conhecer os medos relacionados à doença foi condição para incentivar o autocuidado e alavancar estratégias de enfrentamento, conforme se pode constatar nos seguintes relatos:

Porque eu sei Que, se eu não fizesse, eu sei Que poderia perder meus rins, ficar cega, perder os membros [...] eu não Quero ser uma pessoa... chegar na velhice sem uma perna, ficar cega, eu não Quero isso para mim, então por isso que eu me cuido. [...] (PI0)

[...] Quando eu vejo todo esse povão falando dessas coisas tão perigosas que agora eu tô tendo consciência, eu fico em pânico. (P5)

Percebe-se Que os participantes, apesar de saudáveis, têm medo das complicações futuras e, ao mesmo tempo em Que o medo é um sentimento negativo, pode estimular posicionamentos de autocuidado. Tais medos (da possibilidade de amputações, da autoaplicação de insulina, da extrema dependência de cuidados fornecidos por outros e, no limite, da morte) fazem parte de um processo natural, inerente ao adoecimento, pois são relacionados à nova identidade, Que é conformada com o diagnóstico do $\mathrm{DM}^{(8)}$.

Ao considerarmos os desafios impostos na vida da pessoa Que recebe o diagnóstico e as dificuldades relacionadas à adoção das mudanças inerentes ao tratamento, a demanda por apoio profissional torna-se crescente. Nessa perspectiva, o atendimento em grupo propiciou um clima aberto para livres expressões de idéias e sentimentos, permitindo aos participantes relatarem suas experiências, examinando seus sentimentos mais genuínos e buscando desenvolver seus recursos para a elaboração das emoções mobilizadas ${ }^{(23)}$.

\section{CONSIDERAÇÕES FINAIS}

Diante do objetivo de identificar, entre os participantes do grupo, os sentimentos associados ao diagnóstico do DM, as dificuldades inerentes ao controle glicêmico e as estratégias de enfrentamento adotadas diante das demandas impostas, podemos perceber entre os membros do grupo diferentes modos de lidar com os desafios suscitados pela doença no cotidiano.

O conjunto de dados contribuiu para delimitar categorias Que possibilitaram atender aos objetivos do estudo, à medida Que permitiram construir um panorama dos sentimentos relacionados à doença, bem como descrever possibilidades e limites para o enfrentamento da demanda de cuidados relacionados com seu manejo.

Na categoria "Sentimentos despertados pelo diagnóstico do DM" foram apresentados sentimentos, a maioria com conotação negativa, relacionados ao diagnóstico e tratamento da doença. Nas cat- 
egorias "Dificuldades no controle do DM" e "Estratégias de enfrentamento", a mudança de hábitos foi o fator referido com maior ênfase pelos participantes, cujos relatos apresentaram diferentes posicionamentos frente a tais cuidados, abarcando desde dificuldades, Que na maioria das vezes suscitam baixa adesão, até situações nas Quais é perceptível uma relação mais harmoniosa e estabilizada com tais condições adversas.

Um dado relevante foi o aparecimento da reeducação alimentar como fator mais desafiador para os participantes da pesquisa, o Que evidencia a responsabilidade oue os profissionais de saúde têm de olhar com mais cuidado para essa problemática, Que certamente exige intervenções multiprofissionais para garantir um cuidado mais ampliado, tendo-se em vista a diversidade de fatores relacionados com o processo de alimentação e sua representação no co- tidiano dos participantes e suas famílias.

Ao analisarmos os sentimentos gerados a partir do diagnóstico do DM, a forma como se dá a convivência com a doença no cotidiano, os fatores relacionados com a mudança de hábitos e também a existência de outras demandas de saúde além do manejo do DM, podem-se perceber as principais diferenças Que os participantes apresentam, seja na forma como se colocam diante dos desafios, as estratégias desenvolvidas para lidar com a doença, os apoios disponíveis no contexto familiar e na comunidade, bem como os entraves encontrados. Assim, pode-se inferir Que tais características e posicionamentos assumidos frente às dificuldades influenciam diretamente na adesão ao tratamento e constituem elementos relevantes Que devem ser considerados no planejamento de intervenções mais efetivas.

\section{REFERÊNCIAS}

1. Organização Mundial da Saúde. Cuidados inovadores para condições crônicas: componentes estruturais de ação: relatório mundial. Brasília: Organização Mundial da Saúde; 2003.

2. Thorne S. Chronic disease management: what is the concept? Can I Nurs Res 2008; 40(3): 7-14.

3. Mendes EV. A atenção primária à saúde no SUS. Fortaleza: Escola de Saúde Pública do Ceará; 2002.

4. Silva KL, Sena RR, Grillo MJC, Horta NC, Prado PMC. Educação em Enfermagem e os desafios para a promoção de saúde. Rev Bras Enferm 2009;62(1): 86-91.

5. Sociedade Brasileira de Diabetes. Tratamento e acompanhamento do diabetes mellitus: Diretrizes da Sociedade Brasileira de Diabetes. Rio de Janeiro: Diagraphic; 2007.

6. American Diabetes Association. Standarts of Medical care in diabetes - 2009. Diabetes Care 2009; 32(sup 1): 513-61.

7. Santos MA, Peres DS, Zanetti ML, Otero LM. Grupo operativo como estratégia de atenção integral ao diabético. Rev Enfermagem UERJ 2007; 15(2): 24I-7.

8. Olshansky E, Sacco D, Fitzgerald K, Zickmund S, Hess R, Bryce $\mathrm{C}$, Bryce C, McTigue K, Fischer G. Living with diabetes: normalizing the process of managing diabetes. The Diabetes Educator 2008; 34(6): 1004-12.

9. Pereira GA, Lima MADS. Relato de experiência com grupo na assistência de enfermagem a diabéticos. Rev Gaúcha Enferm 2002; 23(2): 142-157.

10. Minayo MCS. O desafio do conhecimento: pesquisa Qualitativa em saúde. $9^{a}$ ed. São Paulo: Hucitec; 2006.

11. Ministério da Saúde (BR). Conselho Nacional de Saúde. Comitê Nacional de Ética em Pesquisa em Seres Humanos. Resolução 196 de 10 de outubro de 1996: diretrizes e normas regulamentadoras de pesquisa envolvendo seres humanos. Brasília: Ministério da Saúde; 1996.

12. Favoreto CAO, Cabral CC. Narrativas sobre o processo saúdedoença: experiências em grupos operativos de educação em saúde. Interface Comun Saúde Educ 2009; 13(28): 7-1 8.

13. Péres DS, Franco LI, Santos MA. Sentimentos de mulheres após o diagnóstico de diabetes tipo 2. Rev Latino-am enfermagem 2008; 16(1): 101-8.

14. Machado LRC, Car MR. Dialética do modo de vida de portadores de hipertensão arterial: o objetivo e o subjetivo. Rev Esc Enferm USP 2007; 4I(4): 573-80.

15. Peres DS, Santos MA, Zanetti ML, Ferronato AF. Dificuldades dos pacientes diabéticos para o controle da doença: sentimentos e comportamentos. Rev Latino-am Enfermagem 2007; 15(6): $1105-12$

16. Torres HC, Candido NA, Alexandre LR, Pereira FL. O processo de elaboração de cartilhas para orientação do autocuidado no programa educativo em Diabetes. Rev Bras Enferm 2009; 62(2): 312-6.

17. Toral N, Slater B. Abordagem do modelo transteórico no comportamento alimentar. Ciênc Saúde Coletiva 2007; 12(6): $1641-50$

18. Penckofer S, Ferrans CE, Velsor-Friedrich B, Savoy S. The psychological impact of living with diabetes: women day-to-day experiences. Diabetes Educator 2007; 33(4): 680-90.

19. Fischer EB, Thorpe CT, Devellis BM, Devellis RF. Healthy coping, negative emotions, and diabetes management: a systematic review and appraisal. Diabetes Educator 2007; 33(6): 1080-103.

20. Coelho FMC, Pinheiro RT, Horta BL, Magalhães PVS, Garcias CMM, Silva CV. Common mental disorders and chronic noncommunicable diseases in adults: a population-based study. Cad Saúde Pública 2009; 25 (1): 59-67.

21. Thoolen B, Ridder D, Bensing I, Gorter K, Rutten G. Who participates in Diabetes self-management interventions? Issues of recruitment and retainment. Diabetes Educator 2007; 33(3): 465-74.

22. Zanetti ML, Biagg MV, Santos MA, Péres DS, Teixeira CRS. O cuidado à pessoa diabética e as repercussões na família. Rev Bras Enferm 2008; 6I(2): 186-92.

23. Osorio LC. Psicologia grupal: uma nova disciplina para o advento de uma nova era. Porto Alegre: Artmed; 2003. 